Research Paper

\title{
Osteopontin Splice Variants Differentially Exert Clini- copathological Features and Biological Functions in Gastric Cancer
}

\author{
Xiaojian Tang, Jianfang Li, Beiqin Yu, Liping Su, Yingyan Yu, Min Yan, Bingya Liu ${ }^{\bowtie}$, Zhenggang Zhu ${ }^{\bowtie}$ \\ Shanghai Key Laboratory of Gastric Neoplasms, Department of Surgery, Shanghai Institute of Digestive Surgery, Ruijin Hospital, School of \\ Medicine, Shanghai Jiao Tong University, 197 Rui Jin Er Road, Shanghai 200025, People's Republic of China.
}

$\triangle$ Corresponding author: Zhenggang Zhu, MD., PhD. and Bingya Liu, MD., PhD. Shanghai Key Laboratory of Gastric Neoplasms, Department of Surgery, Shanghai Institute of Digestive Surgery, Ruijin Hospital, School of Medicine, Shanghai Jiao Tong University, 197 Rui Jin Er Road, Shanghai 200025, People's Republic of China. Tel: 86-21-64670644; Fax: 86-21-64373909; E-mail: zhuzg@shsmu.edu.cn \& byliu@sjtu.edu.cn.

() Ivyspring International Publisher. This is an open-access article distributed under the terms of the Creative Commons License (http://creativecommons.org/ licenses/by-nc-nd/3.0/). Reproduction is permitted for personal, noncommercial use, provided that the article is in whole, unmodified, and properly cited.

Received: 2012.09.23; Accepted: 2012.12.10; Published: 2012.12.20

\begin{abstract}
Purpose: Gastric cancer (GC) remains a leading cause of death worldwide, and an elevated expression of osteopontin (OPN) may correlate with its poor survival. Alternative splicing of OPN can result in three isoforms, OPN-a, OPN-b and OPN-c. The aim of our current study is to examine the expression pattern and biological functions of OPN splice variants in GC.

Methods: Firstly, we evaluated the expression of OPN splice variants in 7 gastric cell lines, IOI pairs of GC tissues and their adjacent non-tumor tissues by Quantative real-time PCR (QT-PCR). Gain-of-function experiments were subsequently performed to determine their diverse roles in malignant behaviors of GC. Besides, their differential effects on the regulation of crucial downstream molecules were further explored in the anti-apoptotic and pro-metastatic process.

Results: We found that OPN-b is the dominant kind of OPN isoform in GC cell lines. Although the expression levels of three variants were all elevated in GC tissues, increased OPN-b or OPN-c expression could correlate with clinicopathological features. Functional analyses further showed that OPN-b most strongly promoted GC cell survival possibly by regulation of $\mathrm{Bcl}-2$ family proteins and CD44v expressions. Moreover, OPN-c most effectively stimulated GC metastatic activity by increasing secretion of MMP-2, uPa, and IL-8.

Conclusions: Our results suggest that OPN splice variants differentially exert clinicopathological features and biological functions in GC. Therefore, focusing on specific OPN isoform could be a novel direction for developing diagnostic and therapeutic approaches in GC.
\end{abstract}

Key words: OPN splice variants; gastric cancer; clinicopathological feature; biological function; apoptosis; metastasis.

\section{Introduction}

Gastric cancer (GC) is one of the most common malignancies and the leading causes of death worldwide, due to late detection and high recurrence rates [1]. Therefore, understanding the molecular events involved in gastric carcinogenesis will be crucial for the improvement of its diagnosis and therapy.

Osteopontin (OPN) is initially characterized as a glycophosphoprotein secreted by transformed, ma- 
lignant epithelial cells [2]. Previous studies have shown that the elevated expression of OPN is correlated with poor survival in various types of cancers, including GC $[3,4]$. In regards to its biological functions, OPN enhances the survival of cancer cells through its interaction with CD44 variant isoforms $(C D 44 v)$ on the cell surface $[1,5]$, and it can also promote tumor metastasis by increasing expression of matrix metalloproteinase 2 (MMP-2) and urokinase plasminogen activator (uPa) $[6,7]$. Due to the importance of OPN in malignant tumors, further understanding of its role in GC may facilitate the development of prognostic indicators and therapeutic strategies in such patients [1].

OPN precusor-mRNA (pre-mRNA) could be subjected to alternative splicing, leading to full-length OPN-a (i.e., consists of all exons) as well as OPN-b (lacks exon 5) and OPN-c (lacks exon 4) [8]. Recent studies have shown that OPN splice variants are differentially expressed and may have functional heterogeneity in a tumor-specific manner [9]. For example, OPN-b expression dominates in gliomas but is hardly detected in breast cancer tissues [10, 11]. Overexpression of OPN-c promotes tumor metastasis in ovarian cancer but prevents both cell migration and invasion in hepatocellular carcinoma $[12,13]$. Based on these findings, we postulate that specific OPN isoform might become more valuable as cancer target than OPN itself since its diverse roles are dependent on cancer type. However, up to date, no studies have ever focused on three OPN splice variants in GC.

In this study, we aimed to investigate the expression profile and the biological functions of OPN splice variants in GC. Our results demonstrated that only OPN-b and OPN-c are associated with clinicopathological parameters in GC patients, and that OPN isoforms play diverse roles in both anti-apoptotic and pro-metastatic processes.

\section{Materials and methods}

\section{Tissues and Cell Lines}

Our study was approved by the affiliated Ruijin Hospital of Jiaotong University School of Medicine. After written informed consents were obtained from 101 patients diagnosed with GC, the tumor tissues and the adjacent non-tumor tissues were collected during surgery with their clinicopathological data. Human GC cell lines (MKN28, NCL-N87, KATO III, BGC823, SGC7901 and MKN45) and an immortalized gastric epithelium cell line (GES-1) were all grown in RPMI-1640 with $10 \%$ fetal bovine serum (FBS) in a humidified atmosphere of $5 \% \mathrm{CO}_{2}$ at $37^{\circ} \mathrm{C}$.

\section{Plasmid Vector Construction and Transfection of Gastric Cancer Cell Lines}

The full-length cDNA of each OPN isoform was amplified by the same following primers: 5'-CCG CTC GAG ACC ATG AGA ATT GCA GTG ATT TGC T-3'(F) and 5'-CGC GGA TCC TCC TTT TAA TTG ACC TCA GAA GAT GCA C-3'(R). The obtained products were subsequently subcloned into the vector pIRES2-EGFP (BD Biosciences Clontech, Palo Alto, CA, USA). Sequence fidelity and reading frame accuracy of each isoform-overexpressing plasmid were achieved by DNA sequencing analysis. After vector construction, we chose gastric cell lines MKN45 and SGC7901 to respectively transfect with pIRES2-EGFP, pIRES2-OPN-a-EGFP, pIRES2-OPN-b-EGFP and pIRES2-OPN-c-EGFP plasmid, and then cultured them in the medium supplemented with G418 (Promega, Madison, WI, USA) for the establishment of stably transfected cell clones.

\section{Quantitative reverse transcription-PCR (QRT-PCR)}

Total RNA was isolated from the tissues and the cells by using Trizol reagent (Invitrogen, Carlsbad, CA, USA) and then was converted into cDNA by using the Reverse Transcription System (Promega, Madison, WI, USA) with oligo dT. QRT-PCR was carried out with the following sets of primers by using SYBR green PCR master mix (Applied Biosystems, Foster City, CA,USA): OPN-a, 5'-ATC TCC TAG CCC CAC AGA AT- $3^{\prime}(\mathrm{F})$ and $5^{\prime}$-CAT CAG ACT GGT GAG AAT CAT C-3'(R); OPN-b, 5'-ATC TCC TAG CCC CAG AGA C-3'(F) and $5^{\prime}$-AAA ATC AGT GAC CAG TTC ATC AG-3'(R); OPN-c, 5' -TGA GGA AAA GCA GAA TGC TG-3'(F) and $5^{\prime}$-GTC AAT GGA GTC CTG GCT GT-3'(R); CD44v6, 5'-ACG GAA GAA ACA GCT ACC CAG AAG G-3'(F) and 5'-GCT CTC CCT GTT GTC GAA TGG A-3'(R); CD44v7, 5'-CAG CCT CAG CTC ATA CCA GCC A-3'(F) and 5'-ACC TCG TCC CAT GGG GTG TGA-3'(R); GAPDH, 5'-GGA CCT GAC CTG CCG TCT AG-3'(F) and 5'-GTA GCC CAG GAT GCC CTT GA-3'(R). Data were further analyzed by using the comparative $\mathrm{Ct}$ method.

\section{Score of OPN isoform expression}

We first calculated the relative mRNA amounts both in the GC tissues and the adjacent non-tumor tissues. Then we defined the score of OPN isoform expression as previously described [14, 15]: $\log _{\text {e }}$ (the relative mRNA amount of the GC tissue / the relative mRNA amount of the adjacent non-tumor tissue).

\section{Immunofluorescence studies}

As previously described [16], cells were first 
stained with monoclonal anti-OPN antibody (IBL, Japan), and then followed by Cy2- or Cy3-conjugated secondary antibody (Molecular Probes; 1:500 dilution) before examination under fluorescence microscopy. Nuclei were simultaneouly stained by DAPI.

\section{Cell proliferation assays}

Cell proliferation was assessed by water-soluble tetrazolium salt (WST) assays with the Cell Counting Kit-8 (Dojindo, Kumamoto, Japan). And the results were measured as previously described [17]. All experiments were performed in triplicate.

\section{Apoptosis analyses}

The classic oxidative injury model with $\mathrm{H}_{2} \mathrm{O}_{2}$ was established [18]. Cells were treated with $\mathrm{H}_{2} \mathrm{O}_{2}$ $(600 \mu \mathrm{M}$ for MKN45 groups and $300 \mu \mathrm{M}$ for SGC7901 groups) within $30 \mathrm{~min}$ at room temperature. Fifteen hours after withdraw of $\mathrm{H}_{2} \mathrm{O}_{2}$, cells were harvested and assessed for apoptosis by flow cytometry (FACSCalibur, Becton Dickinson,USA). AnnexinV-APC apoptosis kit with propidium iodide (PI) (BD Pharmingen) was used according to the manufacturer's instructions and results were analyzed by CellQuest software (Becton Dickinson). Early and late apoptosis were judged as previously described [19]. All experiments were performed in triplicate.

\section{Western blot analyses}

Cell lysates were separated by SDS-PAGE, then transferred to polyvinylidene difluoridemembranes and probed with the corresponding antibodies: monoclonal anti-OPN which recognized the common epitope of all OPN isoforms (1:500, IBL, Japan); monoclonal anti-phospho-Bcl-2, anti-Bcl-2, anti-Bcl-xl, anti-phospho-Bad, and anti-Bad (1:1000, Cell Signaling Technology, Danvers, MA, USA); monoclonal anti-procaspase-3, and anti-actived-caspase-3 (1:1000, Bioworld Technology, Louis Park, MN, USA). Monoclonal anti-GAPDH (1:5000, Santa Cruz Biotechnology, Santa Cruz, CA, USA) and anti- $\beta$-actin (1:5000, Kangchen, Shanghai, China) were also used as loading controls.

\section{Cell migration and invasion assays}

Cell migration assays were performed by using transwell chambers $(8 \mu \mathrm{m}, 24$-well insert; Corning, Lowell, MA, USA). Cells were added to the upper chamber and incubated with serum-free medium. Medium containing 10\% FBS was added to the lower chamber. After incubation of $48 \mathrm{~h}$, cells which have migrated from the upper chamber to the lower one were stained with crystal violet $(0.1 \%$ in methanol). We then counted the stained cells under inverted mi- croscope and photographed them with 100× magnification. At least three randomly selected fields were counted, and the average number of cells was calculated per field. Invasion assays were completed under the same conditions by using Transwell membranes coated with Matrigel (BD Biosciences).

\section{ELISA Assays}

To analyze the secretion of protein level, culture supernatant of each group was measured with the commercial ELISA kit according to the manufacturer's instructions (Quantikine R\&D Systems, Minneapolis, MD, USA). Briefly, MKN45 cells stably infected with individual OPN isoform or vector control were plated at $5 \times 10^{5}$ cells per well with $1 \mathrm{~mL}$ medium containing $10 \%$ FBS. After incubation of 72 hours, culture supernatant was collected and subjected to ELISA analysis for the measurement of absorbance at $450 \mathrm{~nm}$.

\section{In vivo metastasis assays}

Our animal study was approved by the ethics committee of affiliated Ruijin Hospital of Jiaotong University School of Medicine. For experimental metastasis assays, 4-week-old male nude mice (Shanghai Experimental Animal Center of the Chinese Academy of Sciences, China) were quarantined for a week before tumor implantation. The mice were injected intravenously with $2 \times 10^{6}$ cells of each stably transfected MKN45 group. Livers were collected 8 weeks post injection and photographed for gross morphologic examination as previously described [20].

\section{Statistical analyses}

The Mann-Whitney U-test was used to analyze the expression levels of OPN splice variants in the GC tissues and the adjacent non-tumor tissues. The continuous data were all expressed as mean \pm SE. The Student's $t$ tests were applied when comparisons between two different groups. A p-value $<0.05$ was considered to be statistically significant.

\section{Results}

OPN splice variants presented different expression profiles in gastric cell lines and differentially correlated with clinicopathological features in GC tissues

To investigate the expression pattern of OPN isoforms in GC, we first analyzed their mRNA levels in gastric cell lines, human GC tissues and the adjacent non-tumor tissues. We found that OPN-b was the dominant isoform in six GC cell lines while OPN-a represented the major one in normal gastric cell GES-1. OPN-c was also upregulated in most of GC cell 
lines but barely detected in GES-1 (Fig.1A). However, in human GC tissues, the expression levels of OPN isoforms were all markedly increased than in the adjacent non-tumor tissues ( $\mathrm{P}<0.001$; Fig.1B).

To further determine whether the elevated levels of OPN splice variants are correlated with GC clinicopathological features, 101 GC patients were analyzed. As shown in Table 1, no significant relationship was found between elevated OPN-a expression and any clinicopathological element such as age, gender, tumor site, histological type, lymph node metastasis or TNM stage. However, elevated OPN-b expression in human GC tissues was correlated with larger tumor size $(\mathrm{P}=0.032)$, deeper local invasion $(\mathrm{P}=0.001)$ and advanced TNM stage $(\mathrm{P}=0.044)$. Meanwhile, higher OPN-c level is also related to more lymph node metastasis $(\mathrm{P}=0.001)$, deeper local invasion $(\mathrm{P}=0.012)$ and advanced TNM stage $(\mathrm{P}=0.003)$.
A

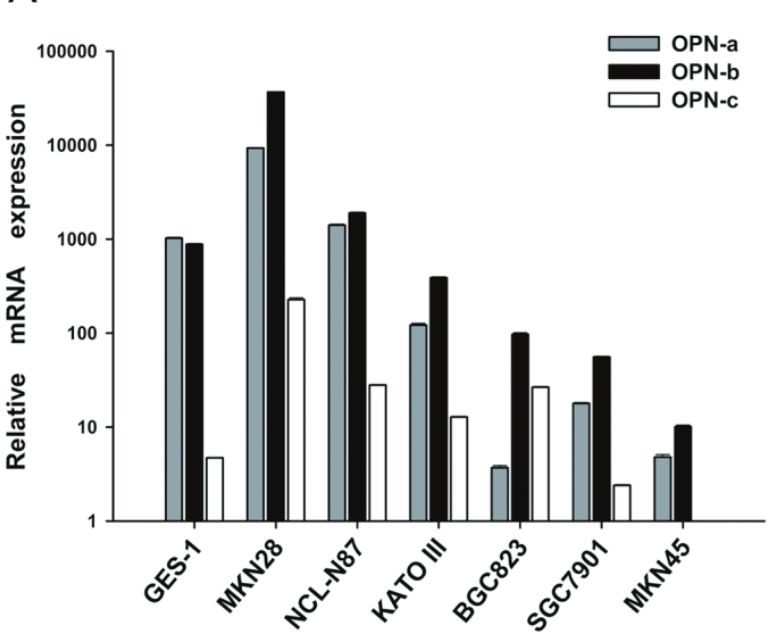

B

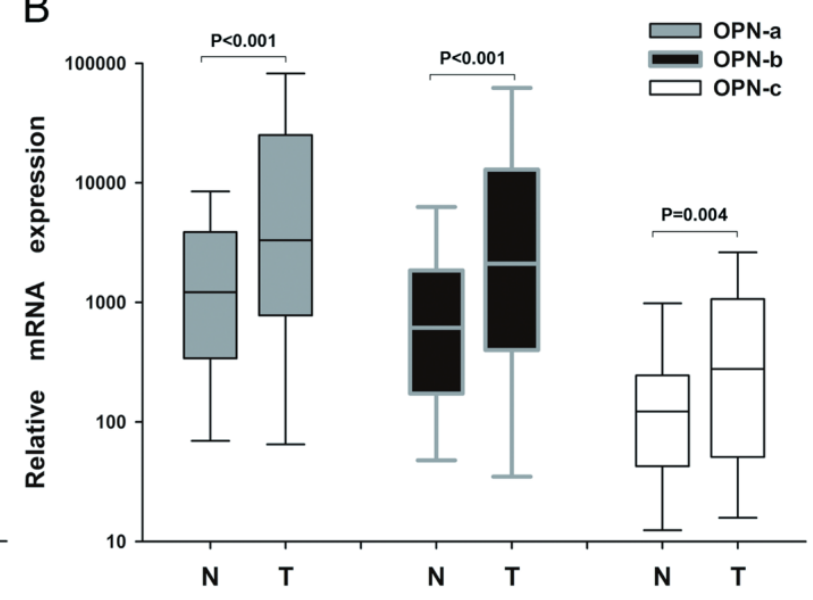

Fig.I Expression pattern of OPN splice variants in GC cell lines and tissues. (A) Relative expression of OPN-a, OPN-b or OPN-c in six GC cell lines and one gastric epithelial cell line (GES-I) as assessed by QRT-PCR assays. (B) Relative expression of OPN-a, OPN-b or OPN-c in I0I pairs of GC tissues and their adjacent non-tumor tissues as assessed by QRT-PCR. Data are shown by box and whisker plot

Table I. Relationship between expression score of OPN transcripts and clinicopathological variables in I0I GC patients.

\begin{tabular}{|c|c|c|c|c|c|c|c|c|}
\hline $\begin{array}{l}\text { Clinicopathological } \\
\text { feature }\end{array}$ & Variable & $\begin{array}{l}\text { No. } \\
\text { case }\end{array}$ & $\begin{array}{l}\text { Score of OPN - a } \\
\text { Expression } \\
(\text { mean } \pm \text { SE })\end{array}$ & $\begin{array}{l}\text { P-Value for } \\
\text { OPN-a }\end{array}$ & $\begin{array}{c}\text { Score of OPN - b } \\
\text { Expression } \\
(\text { mean } \pm S E)\end{array}$ & $\begin{array}{l}\text { P-Value for } \\
\text { OPN-b }\end{array}$ & $\begin{array}{l}\text { Score of OPN - c } \\
\text { Expression } \\
(\text { mean } \pm S E)\end{array}$ & $\begin{array}{l}\text { P-Value } \\
\text { for OPN-c }\end{array}$ \\
\hline \multirow[t]{2}{*}{ Gender } & Male & 76 & $1.23 \pm 0.25$ & 0.935 & $1.19 \pm 0.26$ & 0.680 & $0.87 \pm 0.21$ & 0.255 \\
\hline & Female & 25 & $1.18 \pm 0.51$ & & $1.41 \pm 0.52$ & & $0.34 \pm 0.48$ & \\
\hline \multirow[t]{2}{*}{ Age (years) } & $<60$ & 41 & $1.7 \pm 0.32$ & 0.075 & $1.57 \pm 0.35$ & 0.256 & $1.18 \pm 0.33$ & 0.065 \\
\hline & $\geq 60$ & 60 & $0.89 \pm 0.31$ & & $1.03 \pm 0.30$ & & $0.43 \pm 0.24$ & \\
\hline \multirow[t]{2}{*}{ Location } & Upper & 9 & $0.64 \pm 0.90$ & 0.422 & $0.68 \pm 0.92$ & 0.444 & $0.43 \pm 0.68$ & 0.637 \\
\hline & Lower & 92 & $1.27 \pm 0.23$ & & $1.30 \pm 0.24$ & & $0.77 \pm 0.21$ & \\
\hline \multirow[t]{2}{*}{ Gross type } & Localized & 84 & $1.30 \pm 0.24$ & 0.418 & $1.31 \pm 0.25$ & 0.540 & $0.64 \pm 0.22$ & 0.285 \\
\hline & Infiltrative & 17 & $0.81 \pm 0.64$ & & $0.93 \pm 0.64$ & & $1.21 \pm 0.48$ & \\
\hline \multirow[t]{2}{*}{ Size (maximal diameter) } & $\leq 3 \mathrm{~cm}$ & 26 & $0.61 \pm 0.35$ & 0.113 & $0.41 \pm 0.36$ & $0.032 *$ & $0.18 \pm 0.39$ & 0.103 \\
\hline & $>3 \mathrm{~cm}$ & 75 & $1.43 \pm 0.27$ & & $1.54 \pm 0.28$ & 0.032 & $0.93 \pm 0.23$ & \\
\hline \multirow[t]{2}{*}{ T stage (local invasion) } & pT1-T3 & 33 & $0.85 \pm 0.30$ & 0.204 & $0.28 \pm 0.30$ & $0.001^{* *}$ & $0.12 \pm 0.25$ & $0.012 *$ \\
\hline & pT4 & 68 & $1.39 \pm 0.30$ & & $1.72 \pm 0.29$ & 0.001 & $1.04 \pm 0.26$ & 0.012 \\
\hline \multirow[t]{2}{*}{ Lymph node metastasis } & N0, N1 & 38 & $1.04 \pm 0.38$ & 0.556 & $1.12 \pm 0.39$ & 0.668 & $-0.10 \pm 0.36$ & $0.001^{* *}$ \\
\hline & $\mathrm{N} 2, \mathrm{~N} 3$ & 63 & $1.32 \pm 0.28$ & & $1.33 \pm 0.29$ & & $1.24 \pm 0.21$ & \\
\hline \multirow[t]{2}{*}{ Distant metastasis } & Negative & 97 & $1.19 \pm 0.23$ & 0.499 & $1.21 \pm 0.23$ & 0.363 & $0.70 \pm 0.20$ & 0.398 \\
\hline & Positive & 4 & $1.97 \pm 1.15$ & & $2.28 \pm 1.22$ & & $1.57 \pm 1.18$ & \\
\hline \multirow[t]{2}{*}{ pTNM stage } & I, IIA & 14 & $0.85 \pm 0.43$ & 0.517 & $0.32 \pm 0.44$ & $0.044 *$ & $-0.29 \pm 0.29$ & $0,003 * *$ \\
\hline & IIB, III, IV & 87 & $1.28 \pm 0.25$ & & $1.40 \pm 0.26$ & & $0.90 \pm 0.22$ & \\
\hline
\end{tabular}




\begin{tabular}{lllllllll}
\hline Differentiation & Well-Mod & 35 & $1.57 \pm 0.35$ & 0.250 & $1.68 \pm 0.35$ & 0.175 & $0.86 \pm 0.34$ \\
& Poor & 66 & $1.03 \pm 0.29$ & & $1.02 \pm 0.30$ & & $0.67 \pm 0.25$ \\
Lymphaticvessel inva- & Absence & 72 & $1.04 \pm 0.27$ & 0.220 & $1.09 \pm 0.27$ & 0.288 & $0.58 \pm 0.25$ \\
sion & Present & 29 & $1.65 \pm 0.42$ & & $1.64 \pm 0.43$ & & $1.13 \pm 0.31$ \\
Perineural invasion & Absence & 69 & $1.20 \pm 0.25$ & 0.939 & $1.28 \pm 0.26$ & 0.858 & $0.65 \pm 0.21$ \\
& Present & 32 & $1.24 \pm 0.47$ & & $1.19 \pm 0.48$ & & $0.91 \pm 0.43$ \\
\hline
\end{tabular}

$\left({ }^{*} \mathrm{P}<0.05,{ }^{* *} \mathrm{P}<0.01\right)$.

\section{Overexpression of each OPN splice variant promoted cell proliferation of GC}

To elucidate the contribution of each OPN splice variant in gastric carcinogenesis, each OPN isoform-overexpressing plasmid is constructed and then transfected into both SGC7901 and MKN45 cell lines, in which there were originally low endogenous OPN expression. Stably transfected cell lines were further successfully established with verification of QRT-PCR (Fig.2A and 2B), western blot (Fig.2C) and immunofluorescence (Fig.2D). Also as shown in Fig.2D, predominantly either cytoplasmic or nuclear distribution of OPN protein could be observed in the cells which overexpressed different OPN variants.

We then investigated whether elevated expression of OPN splice variants promoted GC cell growth. In WST assays (Fig. 2E and 2F), SGC7901 and MKN45 cells which overexpressed individual OPN splice variant all enhanced cell proliferation as compared with the corresponding vector control. However, no significant difference of the elevated growth rate was further observed among OPN isoform-overexpressing groups.

\section{Overexpression of OPN-b most strongly promoted GC cell survival by regulation of $\mathrm{Bcl}-2$ family proteins and CD44v expressions}

To further clarify the biological functions of OPN splice variants, we investigated their effects on GC cell survival. After exposure to $\mathrm{H}_{2} \mathrm{O}_{2}$, we found significant decrease of apoptotic rate in GC cells which overexpressed OPN-b or OPN-c as compared with the vector control. However, no such anti-apoptotic effect was observed for OPN-a overexpression (Fig3A). Moreover, OPN-b showed stronger protection from cell apoptosis than that as OPN-c did (Fig. 3B and 3C).

To explore the regulation of essential pro-survival molecules activated by overexpression of individual OPN splice variant, we next focused on Bcl-2 family members which have been demonstrated to play important role in regulating oxidative stress-initiated apoptosis [21]. These proteins can be separated into two groups: pro-survival members such as Bcl-2 and Bcl-xl, and pro-apoptotic members such as Bax and Bad. Besides, phosphorylation of either Bad (p-Bad) or Bcl-2 (p-Bcl-2) can also promote cell survival, thus belonging to pro-survival members $[22,23]$. As we have found in our study (Fig. 3D), among three isoforms, OPN-b could most strongly decrease Bax level, increase p-Bcl-2 expression, reduce degradation of procaspase- 3 and prevent generation of caspase- 3 as compared with the vector control. OPN-c could also strongly upregulate p-Bad expression, moderately decrease Bax level and slightly prevent cleavage of procaspase-3. However, no evident effect was exerted by OPN-a overexpression on the regulation of Bcl-2 family members and consequent caspase- 3 expression.

In gastrointestinal cancer cells, OPN-elicited cell survival can also be facilitated by engagement of its binding receptors CD44v (especially CD44v6 and CD44v7) [5]. To further find out the downstream molecules involved in anti-apoptotic effects, we then focused on the regulation of CD44v6 and CD44v7 expressions triggered by overexpression of individual OPN splice variant. Our investigation found that upregulation of OPN-b or OPN-c, but not OPN-a, could significantly increase both levels of CD44v6 and CD44v7 in MKN45 cells. Furthermore, OPN-b more strongly enhanced CD44v7 expression than that as OPN-c did. (Fig. 3E and 3F)

\section{Overexpression of OPN-c most significantly promoted GC cell migration and invasion by increasing secretion of MMP-2, uPa and IL-8}

We further assessed the effects of OPN splice variants on GC cell migration and invasion. Compared with the vector control (Fig. 4A and 4B), OPN-a-overexpressing MKN45 cells had 1.61 to 1.79 -fold greater migratory capacity $(\mathrm{P}<0.01)$. The similar greater capacity was found by 1.73 to 1.89 -fold for OPN-b-overexpressing MKN45 cells $(\mathrm{P}<0.01)$, and by 2.14 to 2.46-fold for OPN-c-overexpressing MKN45 cells $(\mathrm{P}<0.01)$. Similarly in SGC7901 cells, overexpression of OPN-a, OPN-b and OPN-c could all increase the amount of migrated cells by 1.91 to 2.15-fold, 2.30 to 2.42 -fold, and 2.63 to 2.85 -fold respectively $(\mathrm{P}<0.01)$. 
A

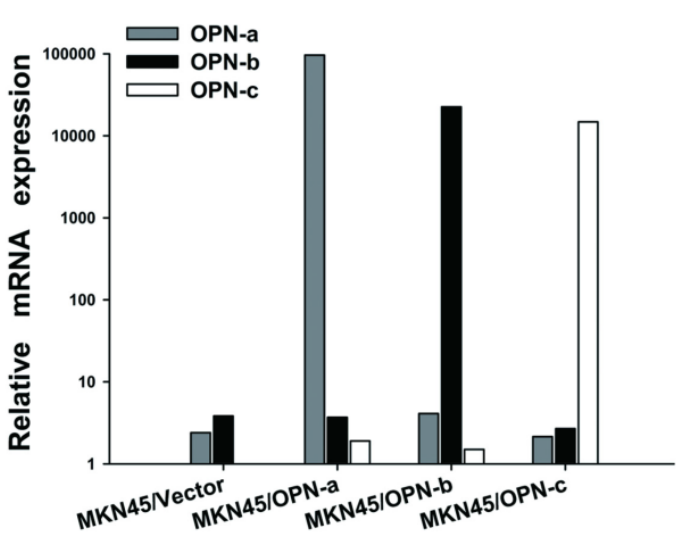

C

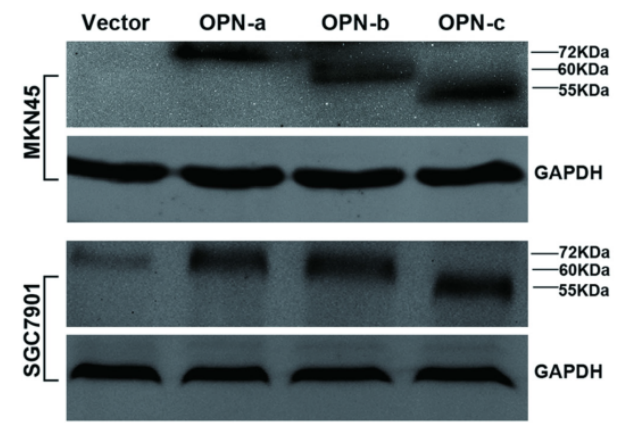

$\mathrm{E}$

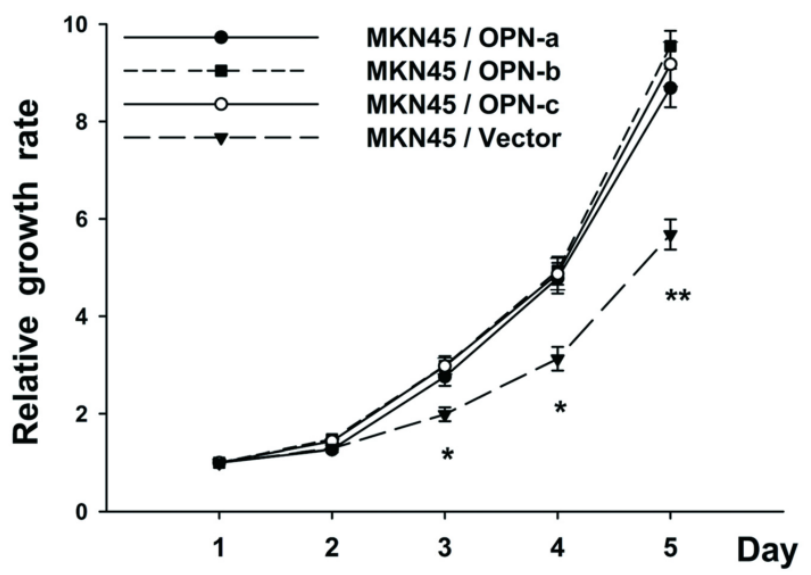

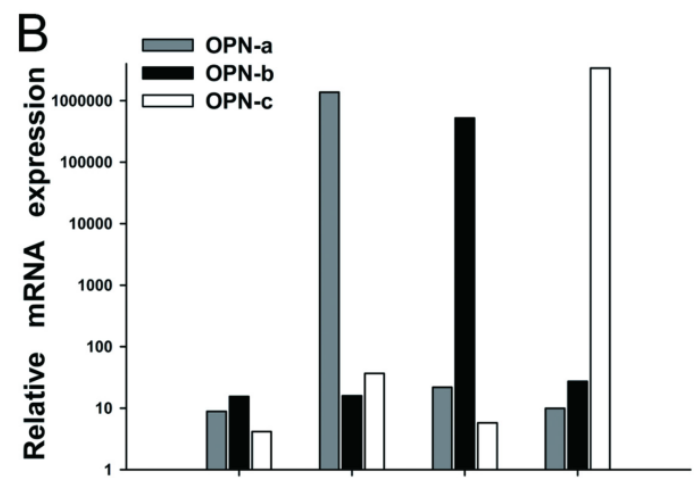

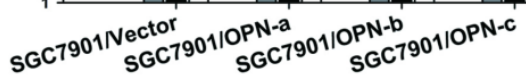

$\mathrm{D}$
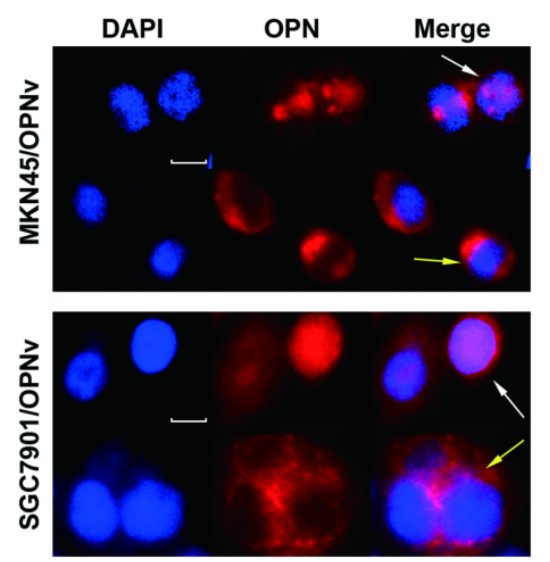

$\mathrm{F}$

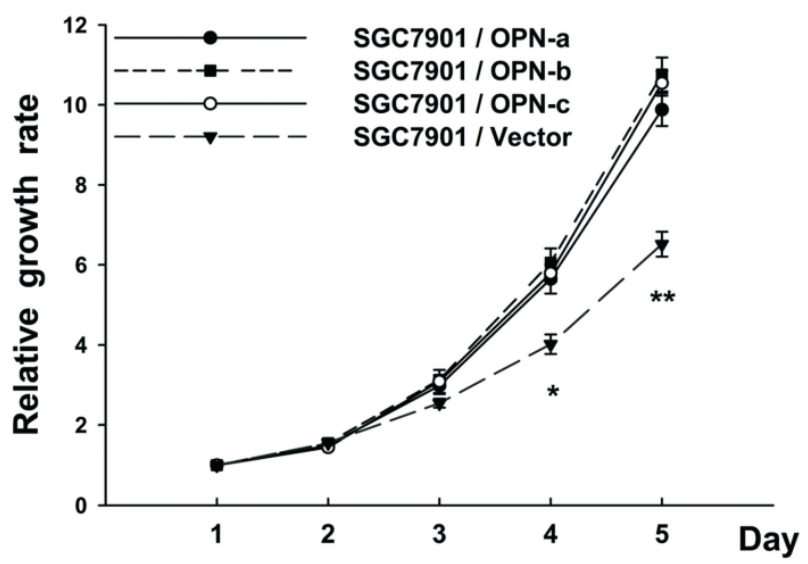

Fig.2 Overexpression of each OPN splice variant promotes GC cell proliferation. (A and B) The ectopic mRNA levels of OPN-a, OPN-b or OPN-c in both MKN45 and SGC 790 I transfectants were detected by QRT-PCR. (C) Overexpressions of OPN-a (around 72kDa), OPN-b (around $60 \mathrm{kDa}$ ) or OPN-c (around $55 \mathrm{kDa}$ ) in two stably transfected cell lines were further confirmed by western blot analyses. GAPDH protein is shown as the loading control. (D) Cells overexpressing individual OPN variant were respectively stained with DAPI (blue) or OPN (red), and two images were further merged. Predominantly either cytoplasmic (yellow arrow) or nuclear (white arrow) fluorescence of OPN protein is presented in both stably transfected cell lines. Scale bar, $10 \mu \mathrm{m}$; OPNv represents OPN splice variant. (E and F) Effects of OPN splice variants on cell proliferation were evaluated by performing WST assays in transfected MKN45 and SGC790I groups. Values represent the mean \pm SE. ( $* \mathrm{P}<0.05, * * \mathrm{P}<0.01$ compared with empty vector transfectants) 
A

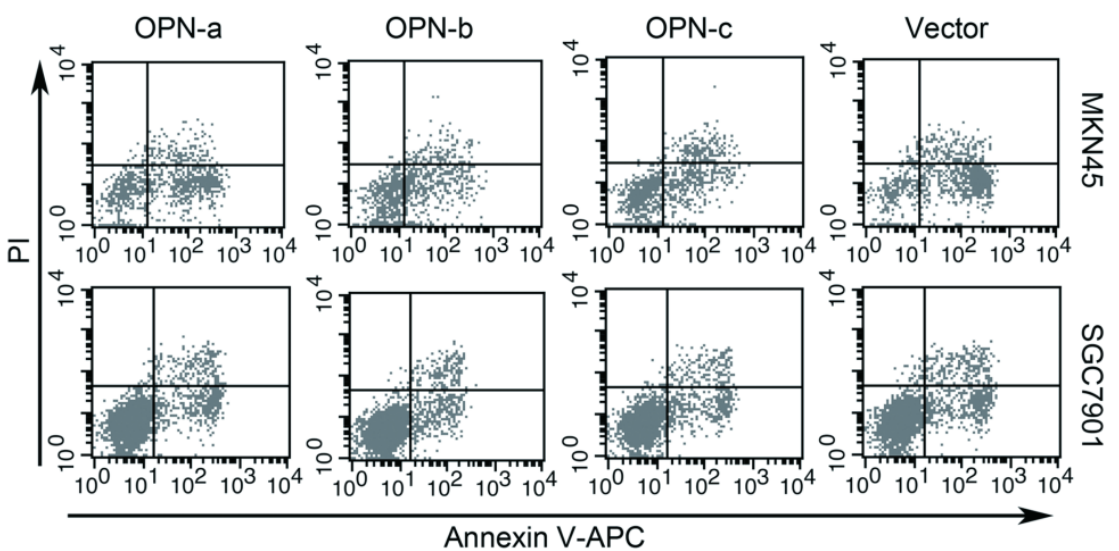

D

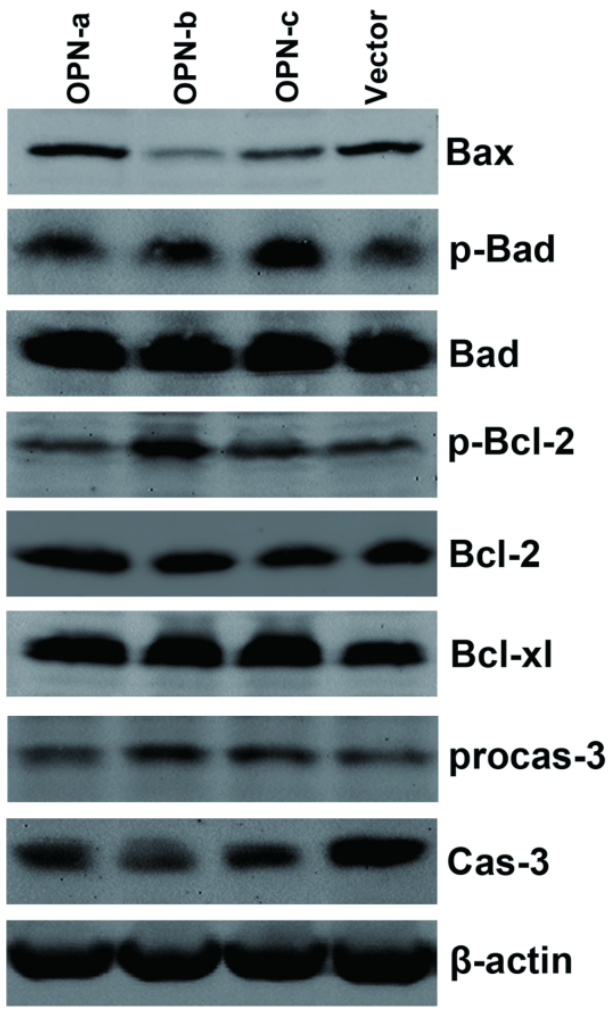

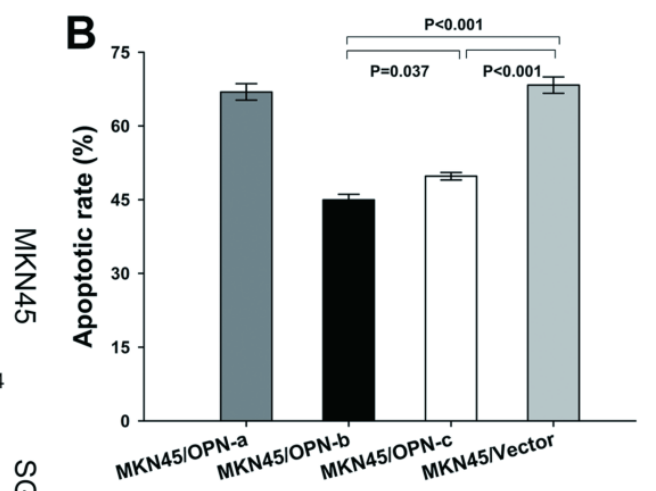

C

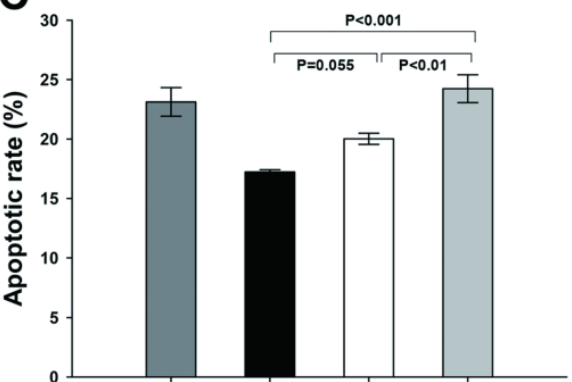

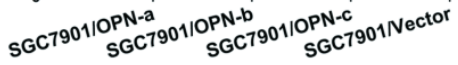

E

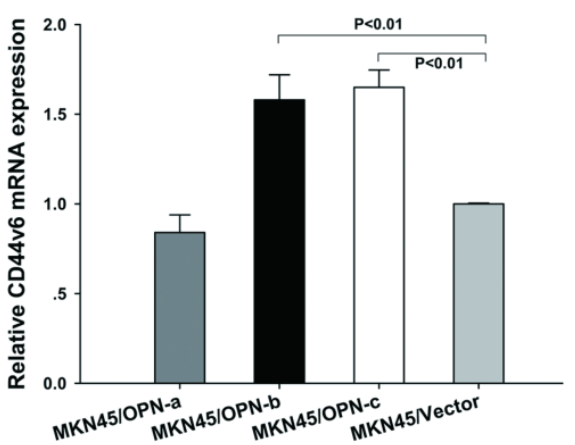

CD44v6

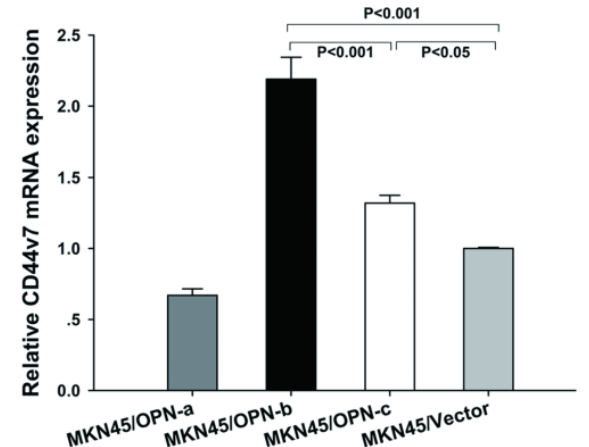

CD44v7

Fig.3 Overexpression of OPN-b most strongly promotes GC cell survival by regulation of Bcl-2 family proteins and CD44v expressions. (A) Representative histograms depict cell apoptosis after stimulus of $\mathrm{H}_{2} \mathrm{O}_{2}$. (B and $\mathrm{C}$ ) Statistically apoptotic rate in each transfected MKN45 and SGC790I group is shown by mean $\pm \mathrm{SE}$ and $\mathrm{P}$ value. (D) Expressions of $\mathrm{Bcl}-2$ family proteins were further assessed by western blot analyses in transfected MKN45 groups. Compared with the vector control, OPN-b most strongly decreased Bax level, increased p-Bcl-2 expression, reduced degradation of procaspase-3 (procas-3) and prevented generation of caspase-3 (cas-3) among three isoforms. OPN-c also strongly upregulated p-Bad expression, moderately decreased Bax level and slightly prevented cleavage of procas-3. (E and F) CD44v6 and CD44v7 levels were additionally analyzed by QRT-PCR in transfected MKN45 groups. Overexpression of OPN-b or OPN-c significantly increased both CD44v6 and CD44v7 levels. Furthermore, OPN-b more strongly upregulated CD44v7 expression than that as OPN-c did. The results are shown as means \pm $S E$ and $P$ values. 
A

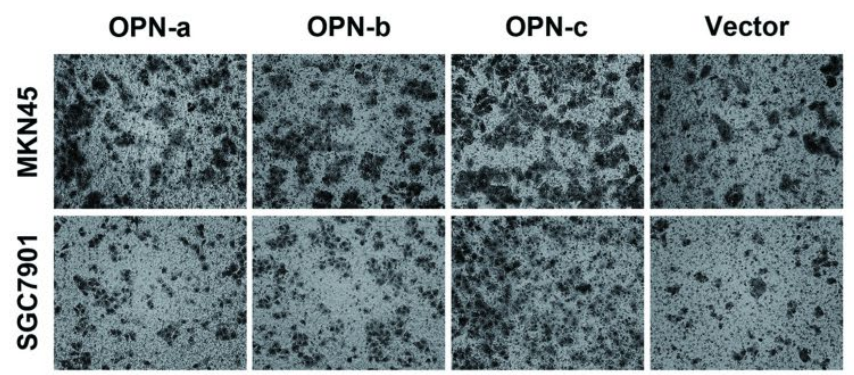

C

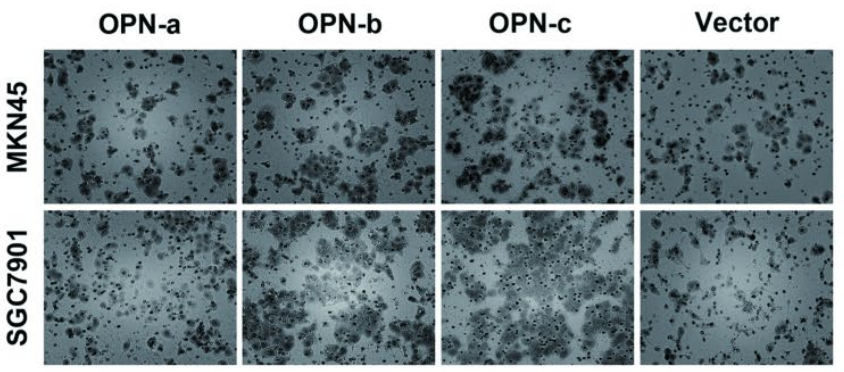

B

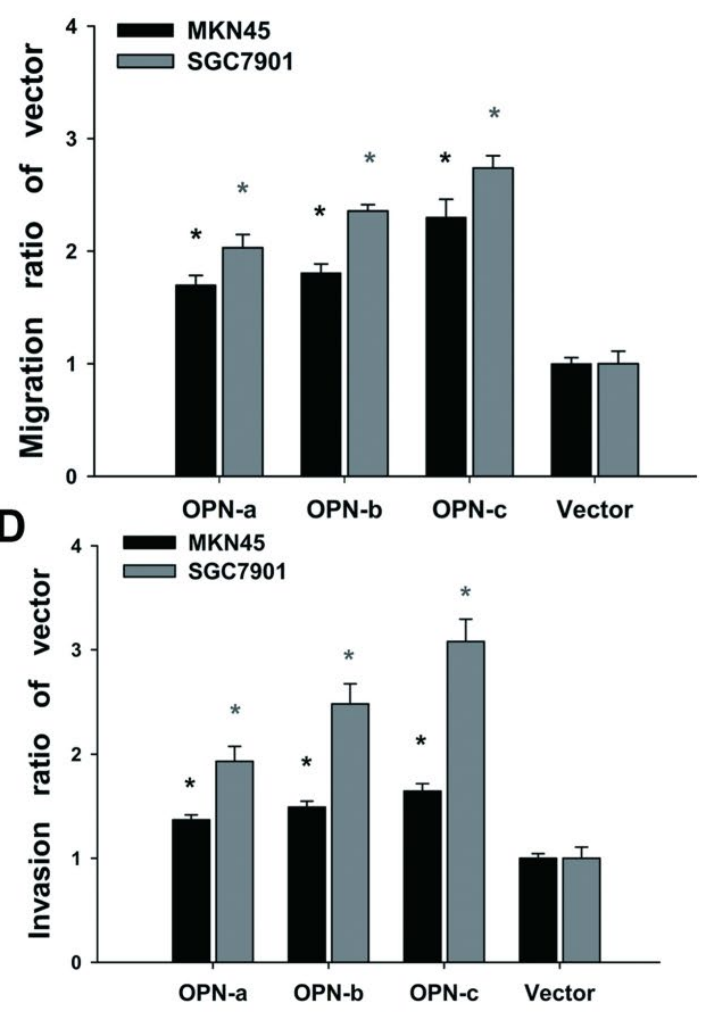

$\mathbf{E}$

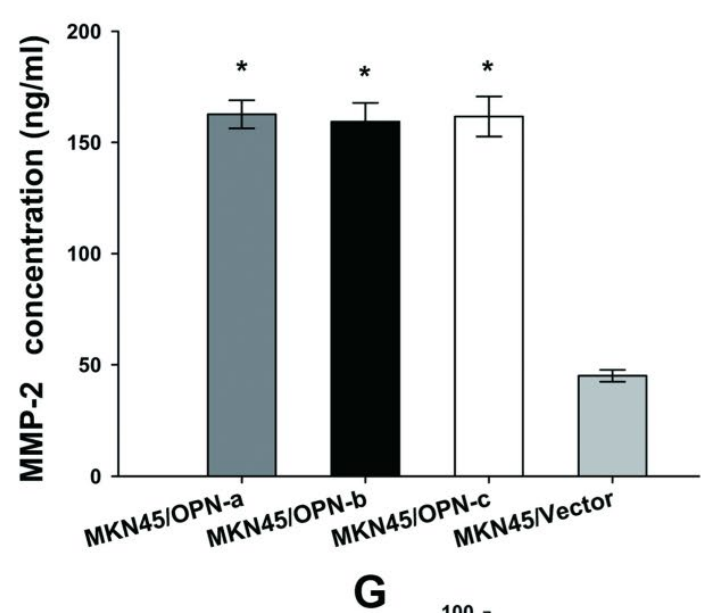

$\mathbf{F}$

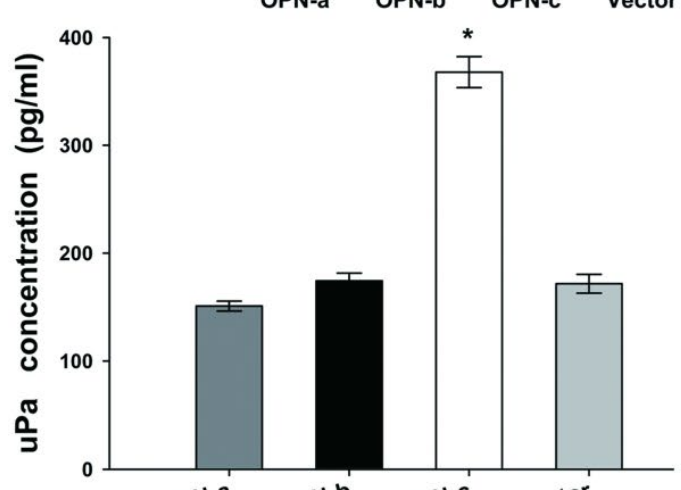

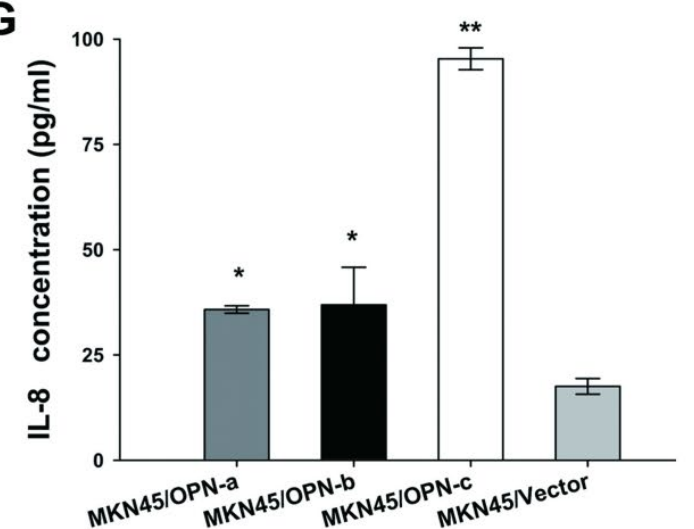

Fig.4 Overexpression of OPN-c most significantly promotes GC cell migration and invasion by increasing secretions of MMP-2, uPa and IL-8. (A and C) Representative photographs of migrated or invasive cells in each transfected MKN45 and SGC790I group. (magnification, 100x) (B and D) Fold-changes of migrated and invasive cells were statistically adjusted to the vector control $(* \mathrm{P}<0.0 \mathrm{O})$. (EF and $\mathrm{G})$ Further detections of MMP2, uPa and IL-8 level in the supernatants of transfected MKN45 groups by ELISA assays. As compared with the vector control, three OPN splice variants increased the secretion of MMP-2 with no difference (Fig 4E, $* \mathrm{P}<0.00 \mathrm{I}$ ), while only OPN-c significantly promoted uPa expression (Fig 4F, $* \mathrm{P}<0.00 \mathrm{I}$ ). Moreover, all variants significantly upregulated IL-8 secretion as compared with the vector control, and OPN-c most strongly exerted this stimulative effect among the three (Fig $4 \mathrm{G}, * \mathrm{P}<0.05, * * \mathrm{P}<0.01$ ). The results are shown as means $\pm \mathrm{SE}$. 
In the invasion assay, ectopic expression of OPN splice variants accordingly promoted cell invasion both in MKN45 cell groups (OPN-a, 1.32 to 1.42-fold increase; OPN-b, 1.43 to 1.55 -fold increase; OPN-c, 1.58 to 1.72-fold increase; $P<0.01$ ) and in SGC7901 cell groups (OPN-a, 1.79 to 2.07-fold increase; OPN-b, 2.29 to 2.67-fold increase; OPN-c, 2.87 to 3.29-fold increase; $\mathrm{P}<0.01$ ) as compared with corresponding vector control. As shown above, OPN-c most significantly promoted both GC cell migration and invasion among three splice variants. (Fig. 4C and 4D)

MMP-2 and $\mathrm{uPa}$ are two key players in the process of degrading extracellular matrix during GC cell migration and invasion [24, 25]. We next analyzed the secretion of MMP-2 and uPa by ELISA assays to investigate their alteration triggered by overexpression of individual OPN splice variant in MKN45 cells. Our data showed that three splice variants all promoted MMP-2 expression ( $\mathrm{P}<0.001$, Fig.4E). However, only OPN-c significantly augmented $\mathrm{uPa}$ secretion as compared with the vector control $(\mathrm{P}<0.001$, Fig. $4 \mathrm{~F})$. IL-8 (CXCL8) is another chemotactic cytokine described to increase invasive behavior of malignant tumor cells [26]. Here, we also focused on the altered secretion level of IL-8 after overexpression of individual OPN isoform. Our results found that all OPN splice variants could increase IL-8 secretion as compared with the vector control, and that OPN-c showed the strongest stimulative effect on IL-8 expression among three isoforms (Fig. 4G).

\section{Overexpression of OPN-b or OPN-c increased in vivo metastatic potential of GC and OPN-c showed the stronger effect}

We further investigated whether ectopic expression of individual OPN splice variant could affect GC metastasis in vivo. Our study in nude mice found that injection of MKN45 cells which overexpressed either OPN-b or OPN-c could significantly develop more liver metastatic nodules as compared with vector control (Fig. 5A). However, no more visible nodules could be observed in mice livers by injection of MKN45 cells which overexpressed OPN-a (Fig. 5A). As statistically shown in Fig. 5B, the average number of liver metastatic nodules was significantly increased in mice injected with GC cells overexpressing OPN-b or OPN-c (OPN-b vs. vector, $72.60 \pm 7.83$ vs. $31.71 \pm$ $3.13, \mathrm{P}<0.001$; OPN-c vs. vector, $145.83 \pm 10.34$ vs. $31.71 \pm 3.13, \mathrm{P}<0.001)$. Moreover, ectopic OPN-c expression in GC cells could more strongly promote in vivo liver metastasis than overexpression of OPN-b did $(\mathrm{P}<0.001$, Fig. 5B).

A

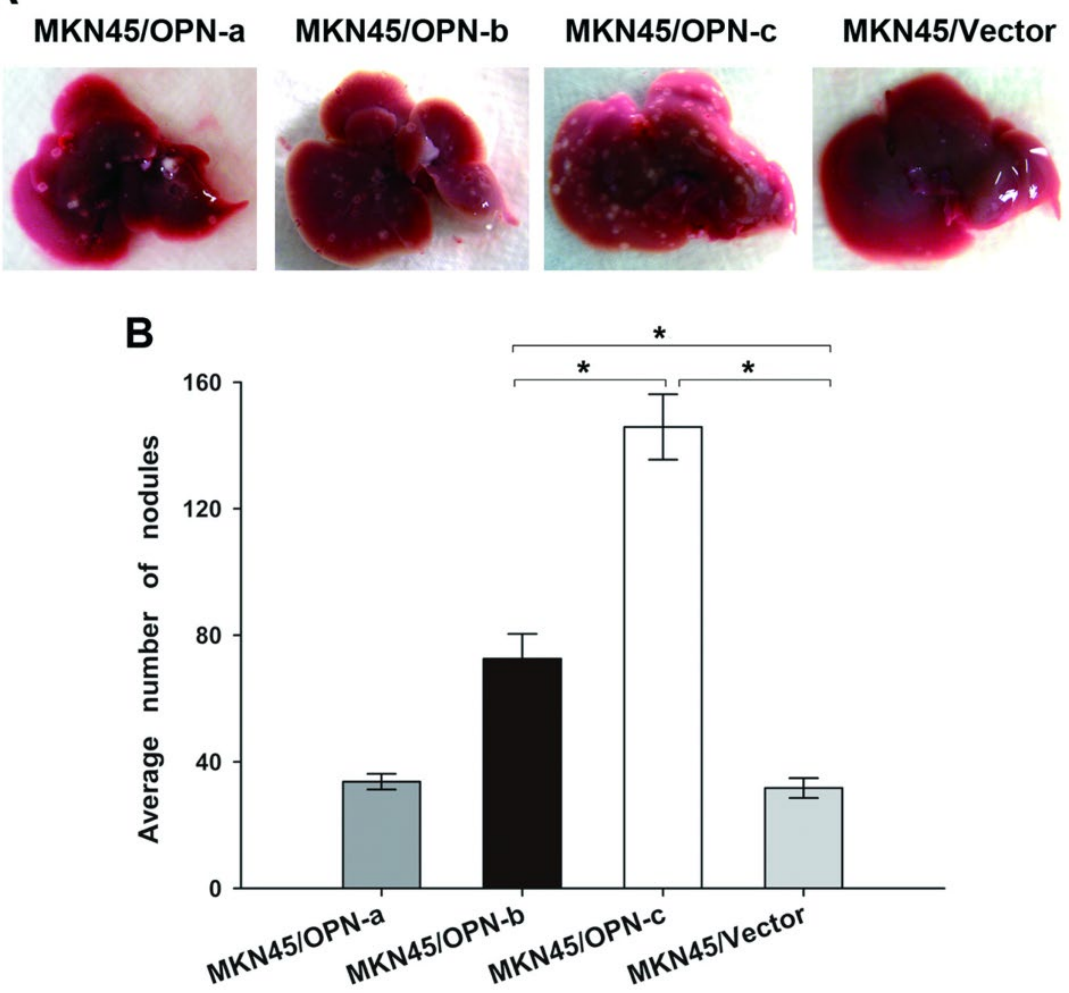

Fig.5 Overexpression of OPN-b or OPN-c increases in vivo metastatic potential of GC and OPN-c shows the stronger effect. (A) Nude mice were injected intravenously with tumor cells of each stably transfected MKN45 group via the lateral tail vein. Livers of nude mice were collected and then photographed to show gross morphologic characteristics 8 weeks post injection. (B) Average numbers of metastatic nodules are statistically analyzed and listed as means $\pm \mathrm{SE}(* \mathrm{P}<0.00 \mathrm{I})$ 


\section{Discussion}

In the present study, we have shown the expression pattern of OPN splice variants in GC. OPN-b was demonstrated as the dominant isoform in GC cell lines, and OPN-c was also elevated in most GC cell lines while barely detected in normal gastric cell line. In GC tissues, increased expression of OPN-b or OPN-c, but not of OPN-a, was associated with diverse clinicopathological features. In according with our observation, it has been reported that high OPN-b or OPN-c expression is correlated with adverse clinicopathological outcomes in both breast cancer and soft tissue sarcoma $[27,28]$. Together with these findings in GC cells and tissues, we therefore postulate that the abnormal alternative splicing which results in elevated OPN-b or OPN-c expression may intensively occur during the progression of GC and then afford it more aggressive potentials.

We next clarified the diverse biological functions of three OPN splice variants through gain-of-function model in which GC cells stably overexpressed individual OPN isoform. We demonstrated that OPN variants presented the same subcellular distribution pattern in predominantly either cytoplasmic or nuclear compartment, which is consistent with the previous report[16]. Besides, we found that three OPN splice variants all increased GC cell proliferation with no significant difference among them, which apparently indicates their similar proliferative competence in GC. After the functional heterogeneity of three OPN isoforms was further observed in both GC anti-apoptosis and metastasis, we also explored their different regulative effect on related downstream targets by overexpressing individual OPN isoform in MKN45 cell line since it presents the lowest endogenous level of each variant among our seven gastric cell lines.

It has been reported that OPN plays important role in preventing programmed cell death in response to pro-apoptotic signals such as oxidative stress stimuli [29]. In agreement with this, our study also demonstrated that both OPN-b and OPN-c could effectively protect GC against cells apoptosis which is induced by $\mathrm{H}_{2} \mathrm{O}_{2}$. Moreover, OPN-b had stronger anti-apoptotic effect than OPN-c did, which is consistent with a previous report of three OPN isoforms in glioma [11]. However, no cell protection was observed in our study by OPN-a overexpression which has also been reported to prevent glioma cell from apoptosis [11]. Therefore, we indicate that the anti-apoptotic abilities of OPN splice variants are diverse in GC. Bcl-2 family members are known as crucial players involved in cell apoptotic process as they are able to regulate the assembly of apoptosome and result in subsequent caspase activation [30]. Our study further showed that OPN-b and OPN-c could both alter the balance between these pro- and antiapoptotic proteins, thus leading to decreased procaspase cleavage and increased cell survival. Notably, OPN-b had an overall stronger pro-survival effect on the modification of this balance than OPN-c did. However, no altered levels of these apoptosis-related proteins could be found by OPN-a overexpression. Our study thus suggests that OPN splice variants exert diverse anti-apoptotic abilities by differently regulating Bcl-2 family proteins. Besides, it has been reported that both CD44v6 and CD44v7 which serve as OPN receptors on cell surfaces can coordinate the activation of downstream anti-apoptotic molecules and protect cancer cell against apoptosis [5, 31, 32]. Accordingly, our results demonstrated that overexpression of either OPN-b or OPN-c could lead to both elevated levels of CD44v6 and CD44v7, thus promoting consequent GC cell survival. Moreover, the most significant upregulation of CD44v7 which resulted from OPN-b overexpression might further explain the strongest anti-apoptotic effect of this isoform since CD44v7 plays a pivotal role in cell survival as previously reported [32].

OPN has been implicated as an important mediator of tumor metastasis [33]. In our study, overexpression of each OPN protein isoform also promoted GC cell migration and invasion, which are two crucial processes in metastasis. It is worth mentioning that OPN-c elicited the strongest pro-metastatic potential among the three variants, thus highlighting its role in GC progression. To further understand the molecular basis involved in the pro-metastasis properties of OPN isoforms, we next detected the secretion of MMP-2 and $\mathrm{uPa}$, both of which are closely related to the metastatic potential of GC [24, 25]. Meanwhile, secretion of IL-8 was explored because it can also act as pro-metastatic chemokine [26]. In our study, ectopic expression of each OPN isoform increased secretion of both MMP-2 and IL-8, with the strongest IL-8 upregulation induced by OPN-c overexpression. However, elevated secretion of uPa could only result from ectopic expression of OPN-c. Overall, our in vitro assays suggest that OPN splice variants all promote GC metastasis through increased MMP-2 and IL-8 secretion, and that OPN-c exerts its most potential pro-metastatic effect among three variants via both elevation of $\mathrm{uPa}$ and strongest upregulation of IL-8. For further in vivo metastasis assays, we demonstrated that both OPN-b and OPN-c, but not OPN-a, promoted hepatic metastasis in nude mice, and that OPN-c also represented the strongest pro-metastatic 
isoform. Those in vivo observations of three OPN splice variants may attribute to the combination of their diverse anti-apoptotic and in vitro pro-metastatic abilities. To our knowledge, this is the first study which has discussed the in vivo metastatic potentials of three OPN isoforms.

Further loss-of-function experiment of three OPN variants would be a valuable complement to the study. However, it's technically difficult for us to downregulate each OPN splice variant both specifically and efficiently by RNA interference method, as the target sequences of each isoform which distinguish the one exclusively from the others were only limited at the specific junction of exons according to the difference among their mRNA structures [8]. Hence, to successfully design loss-of-function study and to possibly attain further therapeutic objective, OPN isoform-specific blocking antibodies need to be established in the future investigation.

As portrayed earlier in this study, there is an aberrant alternative splicing of OPN in the development of gastric neoplasm, which contributes to an aggressive profile of this cancer. Alternative splicing, i.e. the process of pre-mRNA splicing including the excision of introns and ligation of exons, can be activated by the proteins containing serine-arginine-rich sequences (SR proteins) [34]. In gastric tissues, it has been reported that the SR proteins exhibit upregulation and stimulation in response to Helicobacter pylori infection which induces approximately $75 \%$ of gastric cancer cases [35]. Although little is known about the exact mechanisms underlying the alteration in the splice site of OPN pre-mRNA, we suppose that $\mathrm{H}$. pylori infection is involved as original stimulus. Further studies are necessary for the possible correlation between this bacterium contagion and aberrant expression profile of OPN splice variants in GC development.

Furthermore, it is worth exploring the possible mechanism for three OPN splice variants about their distinct functional activity in GC context. As compared with full length OPN (OPN-a), loss of exon 4 $(\mathrm{OPN}-\mathrm{c})$ or exon $5(\mathrm{OPN}-\mathrm{b})$ may result in altered protein structure and thus damage OPN physiological functions in normal gastric tissue. Concretely, two glutamine residues essential for transglutaminase cross-linking are present in sequences corresponding to exon 4. Therefore, OPN-a and OPN-b, but not OPN-c, can form polymeric OPN complexes which increase the stability of protein [36]. However, excision of exon 4 may make OPN-c more soluble and available for integrin receptors, thus resulting in its strong pro-metastatic activity through downstream enhanced signaling [37]. Accordingly, there is one cluster of phosphorylated serine/threonine residues in sequences corresponding to exon 5 which is absent in OPN-b. As previously studied, tumors appear to express hypophosphorylated OPN which may contribute to aggressive cell behaviors [9]. Moreover, total protein phosphorylation status may be not as important as phosphorylation at specific site. Overall, we hypothesize that the phosphorylated residues in exon 5 may be essential to maintain OPN physiological activities, and that the deletion of exon 5 in OPN-b may therefore lead to its strong anti-apoptotic activity. However, further investigation will help elucidate the exact mechanism of altered OPN isoforms' profile and their functional diversity in GC context.

In conclusion, OPN-b is the dominant isoform in GC cell lines and the elevated expression of either OPN-b or OPN-c in GC tissues has their clinicopathological significance. Moreover, OPN-b exerts the strongest anti-apoptotic effect and OPN-c most potentially promotes GC metastasis. Overall, our study suggests that OPN splice variants have different clinical and biological features in GC. Thus, exploring the roles of OPN splice variants in GC will be a novel direction to develop both diagnostic and therapeutic approaches.

\section{Acknowledgements}

This study was supported by grants from National Natural Science Foundation of China (No. 81072012, No. 81172324, No. 30900670, No. 81272749), National High Technology Research and Development Program of China (863 Program, No. 2006AA02A301 and No. 2007AA02Z179), Science and Technology Commission of Shanghai Municipality (No. 10jc1411100, 09DZ1950100, 09DZ2260200, 11jc1407602, and 10140904200), Shanghai Key Discipline (S30204), and Key Projects in the National Science \& Technology Pillar Program of China (No. 2008BA152B03, No. 2011BA203191).

\section{Abbreviations}

GC: gastric cancer;

OPN: osteopontin;

QT-PCR: Quantative real-time PCR;

CD44v: CD44 variant isoforms;

MMP-2 : matrix metalloproteinase 2 ;

$\mathrm{uPa}$ : urokinase plasminogen activator;

pre-mRNA: precusor-mRNA;

WST: water-soluble tetrazolium salt;

GES-1: gastric epithelium cell line;

FBS: fetal bovine serum;

SR proteins: serine-arginine-rich sequences;

p-Bad: phosphorylation of Bad;

p-Bcl-2: phosphorylation of Bcl-2; 


\section{Competing Interests}

The authors have declared that no competing interest exists.

\section{References}

1. Cao DX, Li ZJ, Jiang XO, Lum YL, Khin E, Lee NP, et al. Osteopontin as potential biomarker and therapeutic target in gastric and liver cancers. World J Gastroenterol. 2012; 18: 3923-30.

2. Senger DR, Wirth DF, Hynes RO. Transformed mammalian cells secrete specific proteins and phosphoproteins. Cell. 1979; 16: 885-93.

3. Anborgh PH, Mutrie JC, Tuck AB, Chambers AF. Role of the metastasis-promoting protein osteopontin in the tumour microenvironment. J Cell Mol Med. 2010; 14: 2037-44.

4. Dai N, Bao Q, Lu A, Li J. Protein expression of osteopontin in tumor tissues is an independent prognostic indicator in gastric cancer. Oncology. 2007; 72: 89-96.

5. Lee JL, Wang MJ, Sudhir PR, Chen GD, Chi CW, Chen JY. Osteopontin promotes integrin activation through outside-in and inside-out mechanisms: OPN-CD44V interaction enhances survival in gastrointestinal cancer cells. Cancer Res. 2007; 67: 2089-97.

6. Philip S, Kundu GC. Osteopontin induces nuclear factor kappa B-mediated promatrix metalloproteinase-2 activation through I kappa B alpha /IKK signaling pathways, and curcumin (diferulolylmethane) down-regulates these pathways. J Biol Chem. 2003; 278: 14487-97.

7. Tuck AB, Hota C, Chambers AF. Osteopontin(OPN)-induced increase in human mammary epithelial cell invasiveness is urokinase (uPA)-dependent. Breast Cancer Res Treat. 2001; 70: 197-204.

8. Hijiya N, Setoguchi M, Matsuura K, Higuchi Y, Akizuki S, Yamamoto S. Cloning and characterization of the human osteopontin gene and its promoter. Biochem J. 1994; 303 ( Pt 1): 255-62.

9. Anborgh PH, Mutrie JC, Tuck AB, Chambers AF. Pre- and post-translational regulation of osteopontin in cancer. J Cell Commun Signal. 2011; 5: 111-22.

10. Mirza M, Shaughnessy E, Hurley JK, Vanpatten KA, Pestano GA, He B, et al. Osteopontin-c is a selective marker of breast cancer. Int J Cancer. 2008; 122: 889-97.

11. Yan W, Qian C, Zhao P, Zhang J, Shi L, Qian J, et al. Expression pattern of osteopontin splice variants and its functions on cell apoptosis and invasion in glioma cells. Neuro Oncol. 2010; 12: 765-75.

12. Chae S, Jun HO, Lee EG, Yang SJ, Lee DC, Jung JK, et al. Osteopontin splice variants differentially modulate the migratory activity of hepatocellular carcinoma cell lines. Int J Oncol. 2009; 35: 1409-16.

13. Tilli TM, Franco VF, Robbs BK, Wanderley JL, da Silva FR, de Mello KD, et al. Osteopontin-c splicing isoform contributes to ovarian cancer progression. Mol Cancer Res. 2011; 9: 280-93.

14. Sakakibara T, Hibi K, Koike M, Fujiwara M, Kodera Y, Ito K, et al. Plasminogen activator inhibitor-1 as a potential marker for the malignancy of gastric cancer. Cancer Sci. 2006; 97: 395-9.

15. Wu F, Shirahata A, Sakuraba K, Kitamura Y, Goto T, Saito M, et al. Down-regulation of EGFL8: a novel biomarker for advanced gastric cancer. Anticancer Res. 2011; 31: 3377-80.

16. Junaid A, Moon MC, Harding GE, Zahradka P. Osteopontin localizes to the nucleus of 293 cells and associates with polo-like kinase-1. Am J Physiol Cell Physiol. 2007; 292: C919-26.

17. Feng R, Chen X, Yu Y, Su L, Yu B, Li J, et al. miR-126 functions as a tumour suppressor in human gastric cancer. Cancer Lett. 2010; 298: 50-63.

18. Han $\mathrm{H}$, Long $\mathrm{H}$, Wang $\mathrm{H}$, Wang J, Zhang $\mathrm{Y}$, Wang Z. Progressive apoptotic cell death triggered by transient oxidative insult in $\mathrm{H} 9 \mathrm{c} 2$ rat ventricular cells: a novel pattern of apoptosis and the mechanisms. Am J Physiol Heart Circ Physiol. 2004; 286: H2169-82.

19. Bordon E, Henriquez Hernandez LA, Lara PC, Pinar B, Fontes F, Rodriguez Gallego $C$, et al. Prediction of clinical toxicity in localized cervical carcinoma by radio-induced apoptosis study in peripheral blood lymphocytes (PBLs). Radiat Oncol. 2009; 4: 58.

20. Zhang Y, Pu X, Shi M, Chen L, Song Y, Qian L, et al. Critical role of c-Jun overexpression in liver metastasis of human breast cancer xenograft model. BMC Cancer. 2007; 7: 145.

21. Susnow N, Zeng L, Margineantu D, Hockenbery DM. Bcl-2 family proteins as regulators of oxidative stress. Semin Cancer Biol. 2009; 19: 42-9.

22. Deng X, Gao F, Flagg T, May WS, Jr. Mono- and multisite phosphorylation enhances Bcl2's antiapoptotic function and inhibition of cell cycle entry functions. Proc Natl Acad Sci U S A. 2004; 101: 153-8.
23. Zha J, Harada H, Yang E, Jockel J, Korsmeyer SJ. Serine phosphorylation of death agonist BAD in response to survival factor results in binding to 14-3-3 not BCL-X(L). Cell. 1996; 87: 619-28.

24. Noh S, Jung JJ, Jung M, Kim TS, Park CH, Lim SJ, et al. MMP-2 as a putative biomarker for carcinomatosis in gastric cancer. Hepatogastroenterology. 2011; 58: 2015-9.

25. Okusa Y, Ichikura T, Mochizuki H, Shinomiya N. Urokinase type plasminogen activator and its receptor regulate the invasive potential of gastric cancer cell lines. Int J Oncol. 2000; 17: 1001-5.

26. Strieter RM. Chemokines: not just leukocyte chemoattractants in the promotion of cancer. Nat Immunol. 2001; 2: 285-6.

27. Patani N, Jiang W, Mokbel K. Osteopontin C mRNA expression is associated with a poor clinical outcome in human breast cancer. Int J Cancer. 2008; 122: 2646.

28. Hahnel A, Wichmann H, Greither T, Kappler M, Wurl P, Kotzsch M, et al. Prognostic impact of mRNA levels of osteopontin splice variants in soft tissue sarcoma patients. BMC Cancer. 2012; 12: 131.

29. Chen W, Ma Q, Suzuki H, Hartman R, Tang J, Zhang JH. Osteopontin reduced hypoxia-ischemia neonatal brain injury by suppression of apoptosis in a rat pup model. Stroke. 2011; 42: 764-9.

30. Qiao L, Wong BC. Targeting apoptosis as an approach for gastrointestinal cancer therapy. Drug Resist Updat. 2009; 12: 55-64.

31. Jung T, Gross W, Zoller M. CD44v6 coordinates tumor matrix-triggered motility and apoptosis resistance. J Biol Chem. 2011; 286: 15862-74.

32. Marhaba R, Bourouba M, Zoller M. CD44v7 interferes with activation-induced cell death by up-regulation of anti-apoptotic gene expression. J Leukoc Biol. 2003; 74: 135-48.

33. Wai PY, Kuo PC. Osteopontin: regulation in tumor metastasis. Cancer Metastasis Rev. 2008; 27: 103-18.

34. Karni R, de Stanchina E, Lowe SW, Sinha R, Mu D, Krainer AR. The gene encoding the splicing factor SF2/ASF is a proto-oncogene. Nat Struct Mol Biol. 2007; 14: 185-93.

35. Holland C, Schmid M, Zimny-Arndt U, Rohloff J, Stein R, Jungblut PR, et al. Quantitative phosphoproteomics reveals link between Helicobacter pylori infection and RNA splicing modulation in host cells. Proteomics. 2011; 11: 2798-811.

36. Collighan RJ, Griffin M. Transglutaminase 2 cross-linking of matrix proteins: biological significance and medical applications. Amino Acids. 2009; 36: 659-70.

37. He B, Mirza M, Weber GF. An osteopontin splice variant induces anchorage independence in human breast cancer cells. Oncogene. 2006; 25: 2192-202. 